\title{
Multibeam Antenna Modelling and Blockage Impact Mitigation in 5G Millimeter-Wave Bands
}

\author{
Hamidou Dembélé, Marie Le Bot \\ Radio Networks and Microwaves Dept \\ Orange Labs \\ Cesson-Sévigné, France \\ \{hamidou.dembele,marie.lebot\}@orange.com
}

\author{
François Gallée, Patrice Pajusco \\ Microwaves Dept \\ IMT Atlantique, Lab-STICC, UMR CNRS 6285 \\ F-29238 Brest, France \\ \{francois.gallee,patrice.pajusco\}@imt-atlantique.fr
}

\begin{abstract}
This paper discusses a generic approach proposal for modelling multibeam antenna systems for performance prediction characterized by scan loss and half-power beamwidth. The validation with the existing transmitarrays in the Ka and $\mathrm{V}$ bands is carried out. Using the proposed antenna model, the advantage of multibeam antenna to alleviate the impact of human blockage in millimeter-wave bands is highlighted. Communication performance is evaluated in terms of block error rate through the implementation of the $5 \mathrm{G}$ physical communication chain in a simulation tool.

Index Terms-Multibeam antenna, transmitarray, reflectarray, human blockage, millimeter-wave, $60 \mathrm{GHz}, 5 \mathrm{G}$, block error rate.
\end{abstract}

\section{INTRODUCTION}

Millimeter-wave (mmWave) bands constitute real opportunities for $5 \mathrm{G}$ enhanced mobile broadband (eMBB) applications because of the huge amount of spectrum available in this region of frequencies. Due to the severe propagation loss of electromagnetic waves in mmWave bands, high-gain antenna systems with directional beams are widely deployed to improve the signal-to-noise ratio (SNR) at the receiver $(\mathrm{Rx})$. However, concentrating all energy towards the main propagation path through directional communication eliminates the diversity offered by the multipath and represents a real risk of sudden interruption of communication in the event of obstacles (human or vehicle) in the beam pointing direction [1]. Such a scenario is known as human (or vehicle) blockage phenomenon in mmWaves. To traditionally maintain the communication link on-the-move, the directional beam needs to be mechanically or electronically steered. Mechanical beam steering consists in manually turning the antenna to face the direction of interest. This technique is effective since the antenna gain is maintained and there is a flexibility in the steering range [2], but it is only amenable to static or very slow changing propagation environments. In this way, electronic steering (or beamforming) antennas are proposed for allowing fast beam pointing. Nonetheless, the feed network and electronic constraints are complex and costly in mmWave bands [3] - [4]. In addition, a large number of antennas is required to enhance the system directivity.

To cope with these shortcomings of both electronic and mechanical beam steering, the multibeam antenna (MBA) approach received much attention due to some features such as low profile (small height and width), light weight, cost effective and easy to manufacture [5]. MBAs generally represent an antenna system capable of simultaneously generating a certain number of concurrent and independent directive beams with a high gain to cover a predefined angular range.

To realize an MBA system, several typical approaches have been employed. One approach is to use multiple feed antennas placed at different positions in front of a reflectarray (RA). Another technique is to illuminate one side of a lens or transmitarray (TA) by the incident waves emitted by the feed antennas, while the other side is used to focus the beams in the desired directions. In contrast to RA and TA techniques based on optic principle, MBA design can also be achieved using beamforming circuits such as Butler matrix that can be integrated with an array of antennas into a single substrate [6]. Whatever the design choice, the MBA can be considered as a good compromise in the management of user mobility and especially the phenomenon of blockage in the mmWave context. At first sight, a large beam can simply be used when the main path is blocked by an obstacle, in order to capture energy from the other multipath components; but in this case, a large amount of transmitted power is lost, and the cell coverage is ineffective. Designing an MBA system that simultaneously points in well-chosen directions can solve this issue: if a path is blocked, others are maintained with an efficient distribution of the power.

In this paper, a generic model is proposed for the modelling of the MBA system with the following contributions. Indeed, the proposed model is suitable for the purpose of predicting performance in the design and the hardware realization of an MBA. It is more flexible, complete and realistic for mmWave systems than the antenna models suggested in the standards and others (3GPP [7], ITU-R [8], DuFort [9]) for which the gain reduction for a certain scan range is tolerated. This model is then integrated in a simulation chain of the $5 \mathrm{G}$ physical layer, including multipath propagation channels and human blockage. Simulations are run to show the advantage of the MBA.

The rest of the paper is organized as follows. In section II, the proposed model is described with the input parameters as well as the assumptions. Section III is focused on the application of the model and its validation with existing MBA 
TAs. Section IV is devoted to the link level evaluation in the context of human blockage. Conclusion and future work are given in section $\mathrm{V}$.

\section{Multibeam Antenna Model Proposal}

Typically, MBA systems are made up of a finite number of input ports, each connected to an antenna called feed antenna. The excitation of each port allows to control a single beam pointing at a predefined direction [6]. Multiple beams pointing in different directions can be transmitted simultaneously from a shared aperture to cover a certain angular range when several ports are excited. In general, the MBA performance is characterized by the scan range which leads to the reduction in antenna gain called scan loss (SL). Other parameters such as aperture efficiency, sidelobe level (SLL), beamwidth can also be used.

\section{A. Study Assumptions and General Expression}

In the development of the MBA model, some assumptions were taken into account. First of all, based on the RA and TA structures, we generally consider the MBA system as a two-dimensional planar aperture antenna illustrated in Fig. 1. We assume that the radiation in both perpendicular plans xoy and zox are not dependent, and the waves on the antenna aperture are in phase due to the phase correction performed by the unit-cells (transmitters) of the RAs and TAs. In this case, only the amplitude distribution of the field is considered, from which the radiation pattern at the antenna broadside is defined. Assuming that each of the feed antennas illuminates the center of the radiating surface, the beam patterns in other directions are estimated by the surface projection. However, the model does not depend on the number of unit-cells, but rather on the dimensions of the aperture. And the increase in the level of sidelobes when the scan range becomes wide is not also taken into account. Consequently, the general expression of the antenna gain pattern in the desired pointing direction $\left(\theta_{0}, \phi_{0}\right)$ around the antenna broadside is given by,

$$
G(\theta, \phi)=\left(\frac{G_{0}}{S L\left(\theta_{0}, \phi_{0}\right)}\right) g\left(\theta-\theta_{0}, \phi-\phi_{0}\right),
$$

where $\theta$ and $\phi$ represent the angles of elevation and azimuth, respectively. $G_{0}$ is the antenna gain at broadside, $S L\left(\theta_{0}, \phi_{0}\right)$ defines the scan loss value in the beam direction $\left(\theta_{0}, \phi_{0}\right)$ and $g\left(\theta-\theta_{0}, \phi-\phi_{0}\right)$ is the normalized gain radiation pattern. The scan loss $S L$ is referenced to the antenna broadside.

\section{B. Antenna Gain at broadside}

To reduce the complexity of the calculation of the antenna directivity, several approximate expressions are proposed in the literature, among which the Kraus formula given by [13],

$$
D_{0} \approx \frac{41253}{\theta_{H P B W_{0}} \cdot \phi_{H P B W_{0}}},
$$

is suitable for antenna systems with narrow beams and very low SLLs. $D_{0}$ is the antenna broadside directivity from which the gain $G_{0}$ is naturally defined by,

$$
G_{0}=\eta D_{0},
$$

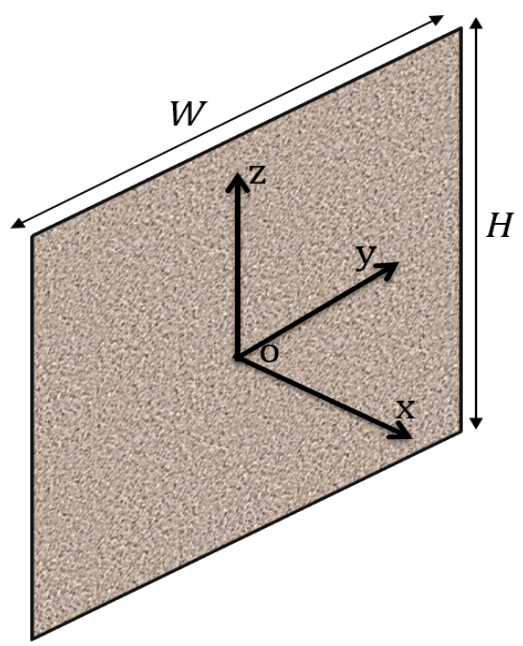

Fig. 1. Rectangular aperture with dimensions $W$ and $H$.

with $\eta$ the antenna efficiency.

\section{Normalized Radiation Pattern and Scan Loss}

The normalized gain radiation pattern at the antenna broadside is defined by,

$$
g(\theta, \phi)=|A(\theta, \phi)|^{2},
$$

where $A(\theta, \phi)$ represents the two-dimensional field radiation pattern. Using Goudet's formula [11] in which we assumed that the radiations in the two perpendicular planes are not dependent and waves on the antenna aperture are in phase, $A(\theta, \phi)$ can be expressed as,

$$
A(\theta, \phi)=A(\theta) \cdot A(\phi),
$$

with $A(\theta)$ and $A(\phi)$ the field radiations in elevation and azimuth defined by (6) and (7), respectively.

$$
\begin{aligned}
A(\theta) & =\frac{1+\cos (\theta)}{2} S F(\theta, H, \lambda) . \\
A(\phi) & =\frac{1+\cos (\phi)}{2} S F(\phi, W, \lambda) .
\end{aligned}
$$

$S F(\theta, H, \lambda)$ and $S F(\phi, W, \lambda)$ define the spatial Fourier transform of the electric field in elevation and azimuth, respectively. $H$ and $W$ are the antenna aperture dimensions as illustrated in Fig. 1 and $\lambda$ the wavelength. Consequently, the normalized radiation pattern in the direction $\left(\theta_{0}, \phi_{0}\right)$ is first obtained by replacing $\theta$ and $\phi$ by $\theta-\theta_{0}$ and $\phi-\phi_{0}$ in the above expressions. And then the aperture size $H$ must be replaced by the equivalent size $H_{\text {equiv }}=H \cdot \cos \left(\theta_{0}\right)$ in elevation and the size $W$ by $W_{\text {equiv }}=W \cdot \cos \left(\phi_{0}\right)$ in azimuth due to the projected area reduction which is estimated by a cosine reduction of effective aperture surface for the scan directions $\theta_{0}$ and $\phi_{0}$ [12]. In this way, the beamwidth for the scan angles increases from the antenna broadside and is characterized by the half-power beamwidth (HPBW) in elevation by,

$$
\theta_{H P B W}\left(\theta_{0}\right)=\frac{\theta_{H P B W_{0}}}{\cos \left(\theta_{0}\right)}
$$


and in azimuth by,

$$
\phi_{H P B W}\left(\phi_{0}\right)=\frac{\phi_{H P B W_{0}}}{\cos \left(\phi_{0}\right)},
$$

where $\theta_{H P B W_{0}}$ and $\phi_{H P B W_{0}}$ represent the HPBWs at the antenna broadside in elevation and azimuth, respectively. As the beams are scanned in both perpendicular plans independently, the scan loss in the beam direction in elevation $\theta_{0}$ can be approximated in $\mathrm{dB}$ units by,

$$
S L\left(\theta_{0}\right)=-20 \log _{10}\left(\left|\cos \left(\theta_{0}\right)\right|\right) .
$$

Likewise, if the scan is performed in the azimuth plane, the scan loss is defined by,

$$
S L\left(\theta_{0}\right)=-20 \log _{10}\left(\left|\cos \left(\phi_{0}\right)\right|\right) .
$$

With the above expression, it can be noted that the $S L$ does not depend on the frequency and dimensions of the antenna unlike HPBWs.

\section{Application And VAlidation of the Model}

The purpose of this section is to highlight the application of the proposed method and the performance comparison in terms of beamwidth and scan loss with some existing MBA TAs. To this end, parameters such as frequency, TA dimensions, antenna efficiency and field amplitude distribution on the antenna aperture are required. Using the procedure explained in section II-C, $g(\theta, \phi)$ can be expressed as,

$$
g(\theta, \phi)=g(\theta) \cdot g(\phi),
$$

with $g(\theta)$ and $g(\phi)$ the radiation patterns in both planes.

The choice of the distribution really impacts the beamwidth and the SLL. White [14] mentioned that better sidelobe performance can be achieved by MBA systems with cosine or cosine-squared aperture distributions. Nonetheless, any other distribution could be used depending on one's goal.

By considering the narrow-beam approximation since the beginning, and adopting the normalized angle coordinate $u=$ $\frac{\pi H}{\lambda} \sin \theta$, radiation characteristics [15] in elevation are summarized in Table I for cosine and cosine-squared distributions. Similar expressions are obtained in azimuth by replacing $\theta$ by $\phi, H$ by $W$ and the angle coordinate $u$ by $v=\frac{\pi W}{\lambda} \sin \phi$. In this work, the choice criterion of a distribution is mainly based on the value of HPBW and eventually the SLL.

In the validation procedure of the proposed approach, several models of existing antennas with different frequency bands were used. But, in this paper, we have only focused on the $\mathrm{Ka}$ and $\mathrm{V}$ bands when it comes to mmWave communication systems.

\section{A. Performance Comparison with Ka-Band TA}

For comparison with Di Palma's electronically reconfigurable TA [16], which is composed of a squared surface of dimension $\approx 100 \mathrm{~mm}$ at $29 \mathrm{GHz}$ and a boresight $H P B W=$ $7.5^{\circ}$, the cosine aperture distribution was assumed. With these parameters, a performance comparison is performed as follows. Fig. 2 shows the scan loss with respect to the
TABLE I

RADIATION CHARACTERISTICS

\begin{tabular}{|c|c|c|c|}
\hline $\begin{array}{c}\text { Aperture } \\
\text { Distribution }\end{array}$ & Radiation Pattern $g(\theta)$ & $\begin{array}{c}\theta_{H P B W_{0}} \\
{[\mathrm{deg}]}\end{array}$ & SLL [dB] \\
\hline Cosine & $\left|\frac{1+\cos (\theta)}{2} \frac{(\pi / 2)^{2} \cos (u)}{(\pi / 2)^{2}-u^{2}}\right|^{2}$ & $68.8 \frac{\lambda}{H}$ & -23.2 \\
\hline $\begin{array}{c}\text { Cosine- } \\
\text { Squared }\end{array}$ & $\left.\frac{1+\cos (\theta)}{2} \frac{\sin (u)}{\left(1-(u / \pi)^{2}\right) u}\right|^{2}$ & $83.2 \frac{\lambda}{H}$ & -31.5 \\
\hline
\end{tabular}

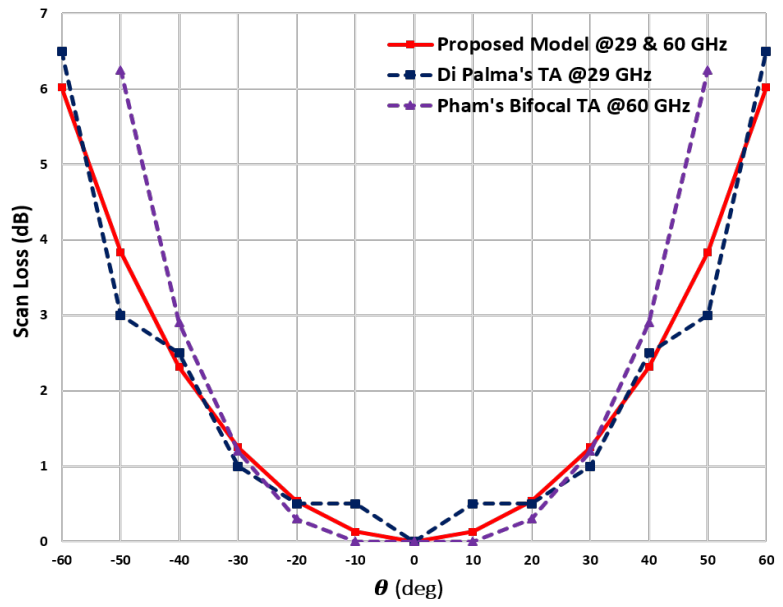

Fig. 2. Scan loss performance at 29 and $60 \mathrm{GHz}$.

directions of the beams. We can note a quite good agreement between the proposed model which can be seen as a prediction model and the measurement results performed by Di Palma et al. for a scan range of $\pm 60^{\circ}$ off broadside. In Fig. 3, the beamwidth comparison is highlighted for the same scan range. We also notice that both beamwidth curves achieve similar performance in the same scan range as for the scan loss.

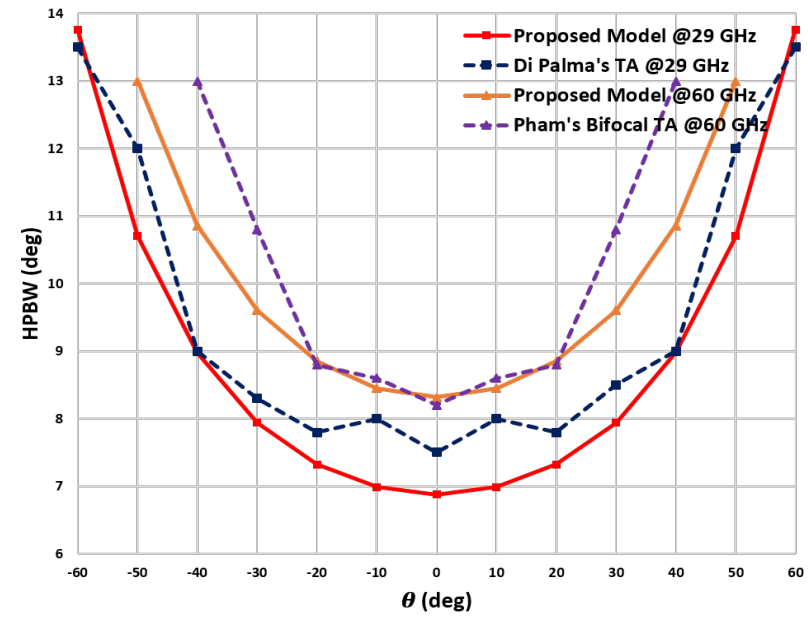

Fig. 3. Beamwidth performance at 29 and $60 \mathrm{GHz}$. 


\section{B. Performance Comparison with V-Band Bifocal TA}

In this subsection, the bifocal TA physically realized by Pham et al. [17] is used in the validation procedure at $60 \mathrm{GHz}$. The system is composed of a squared surface of dimension 50 $\mathrm{mm}$ and a $H P B W=8.2^{\circ}$ is achieved at the broadside.

By considering a cosine-squared distribution in addition to system parameters, the scan loss and beamwidth performance versus the beam directions are given in Figs. 2 and 3, respectively. Comparing the proposed model with Pham's bifocal TA measurements, we notice similar performance for a scan range of $\pm 35^{\circ}$ around the antenna broadside and a difference of about $1.5 \mathrm{~dB}$ at $\pm 45^{\circ}$ in terms of scan loss. Outside the $\pm 45^{\circ}$ angular sector, the discrepancies become significant. This may be justified by the spill-over radiation and hardware component loss that are not taken into account in the proposed prediction model, especially at high frequencies.

\section{Link LEVEl Simulation AT $60 \mathrm{GHz}$}

In this section, the link level performance evaluation is performed in order to first show the impact of human blockage on $5 \mathrm{G}$ quality of service (QoS) in terms of block error rate (BLER). And then, the advantage of the MBA system using the proposed model in the context of blockage is highlighted. In this way, the end-to-end data processing of the $5 \mathrm{G}$ physical communication chain shown in Fig. 4 is used. This $T x / R x$ chain is mainly composed of key blocks such as cyclic redundancy check (CRC), low-density parity-check (LDPC) channel coding, orthogonal frequency-division multiplexing (OFDM) modulation, clustered delay line (CDL) channel and minimum mean square error (MMSE) equalizer.

\section{A. Human Blockage Modelling}

Influence of human movement between the $\mathrm{Tx}$ and $\mathrm{Rx}$ constitutes a critical aspect of the mmWave propagation. In several experimental studies, measurement results have shown that human body has a significant impact on the signal strength in mmWave bands. The IEEE 802.11ad $60 \mathrm{GHz}$ channel modelling mentions that human shadowing is in the range of 18 to $36 \mathrm{~dB}$ [19]. Collonge et al. [20] have also mentioned an attenuation of $20 \mathrm{~dB}$ when the line-of-sight (LOS) path is shadowed by a person at $60 \mathrm{GHz}$. In fact, in the specification defined by 3GPP, the human body is modelled by a blocking region with an angular space $\Delta \theta$ centered around $\theta_{c}$ in elevation and another $\Delta \phi$ centered around $\phi_{c}$ in azimuth as illustrated in Fig. 5. With this approach, the principle is to attenuate the power of any path whose arrival angles fall in the blocking region, and the attenuation value is given by a knife-edge diffraction formula. With the highest values of blocker parameters, i.e $\Delta \theta=15^{\circ}, \Delta \phi=45^{\circ}$ and the distance from the blocker to the $\operatorname{Rx} r=2 m$ [7], an attenuation of 16 $\mathrm{dB}$ at $60 \mathrm{GHz}$ is deduced from the 3GPP non-self-blocking model. The non-self-blocking is characterized by the presence of humans or vehicles moving around the user as shown in Fig. 6. Although this model is used for the illustration and simulation purpose in this work, the values of 3GPP human shadowing may be subject to criticism. The difference in the human body attenuation when comparing the 3GPP modelling with other values [19] - [20], could be justified by the choice of the antenna system in the experiments. In other words, the values are realistic and significant when the directional antenna is used for the human shadowing assessments. In this case, the communication link can be interrupted. However, as explained by Raghavan et al. [21], the 3GPP standard assumes an omnidirectional antenna for the human blockage modelling, which leads to low attenuation values.

\section{B. Simulation Scenarios}

Using the 3GPP human blockage model at $60 \mathrm{GHz}$, three simulation scenarios were considered in this work as follows.

1) Scenario 1: In this first scenario, there is no blocker between the Tx and Rx, and a single communication beam is used and steered towards the main propagation path, i.e the path with the highest power.

2) Scenario 2: The second scenario is exactly scenario 1 with the presence of a human blocker located in the direction of the single beam.

3) Scenario 3: The third scenario can be seen as the solution to scenario 2 with the application of the multibeam aspect. Since blockage occurs in the direction of the first beam, an additional beam is used, and points to the propagation path with the second highest power.

\section{Simulation Results}

The simulation parameters are summarized in Table II. The 3GPP CDL-C [7] channel model with 24 propagation paths was used. In the scenarios, the proposed MBA is only used at the base station (Tx) with the broadside gain of $20.3 \mathrm{dBi}$ from the parameters of the $60 \mathrm{GHz}$ bifocal TA of Pham et al. [17]. An omnidirectional antenna with a $0 \mathrm{dBi}$ gain is used at the user equipment $(\mathrm{Rx})$ level for simplicity.

In Fig. 7, a comparison of the performance in terms of BLER versus SNR of the three scenarios is highlighted for a fixed throughput of $1.5 \mathrm{Gbits} / \mathrm{s}$. This data rate is given by the modulation and coding scheme (MCS) 15 [23], i.e 64QAM modulation with a code rate of 0.65 for a bandwidth of 400 $\mathrm{MHz}$ [24]. Simulation results reveal that to ensure the same $5 \mathrm{G}$ eMBB QoS, scenario 2 with human blocking in the single beam direction requires a $16 \mathrm{~dB}$ increase in SNR with respect to scenario 1 in which there is no human blockage. However, we only notice a difference of $2.8 \mathrm{~dB}$ between scenario 1 and scenario 3. Indeed, in scenario 3 , using an additional beam that points towards the second propagation path when the first beam is blocked, almost guarantees the same QoS with the same SNR values and avoids the link interruption between the Tx and the Rx.

With these results, the blockage problem has been analyzed through the SNR variations for a fixed target throughput. Nevertheless, similar performance can be achieved in the three scenarios with the same SNR values as shown in Fig. 8, with a variation of throughputs as follows. Compared to ideal scenario 1, the communication data rates in scenario 2 must be drastically reduced from $1.5 \mathrm{Gbits} / \mathrm{s}$ to $227.9 \mathrm{Mbits} / \mathrm{s}$ due to 


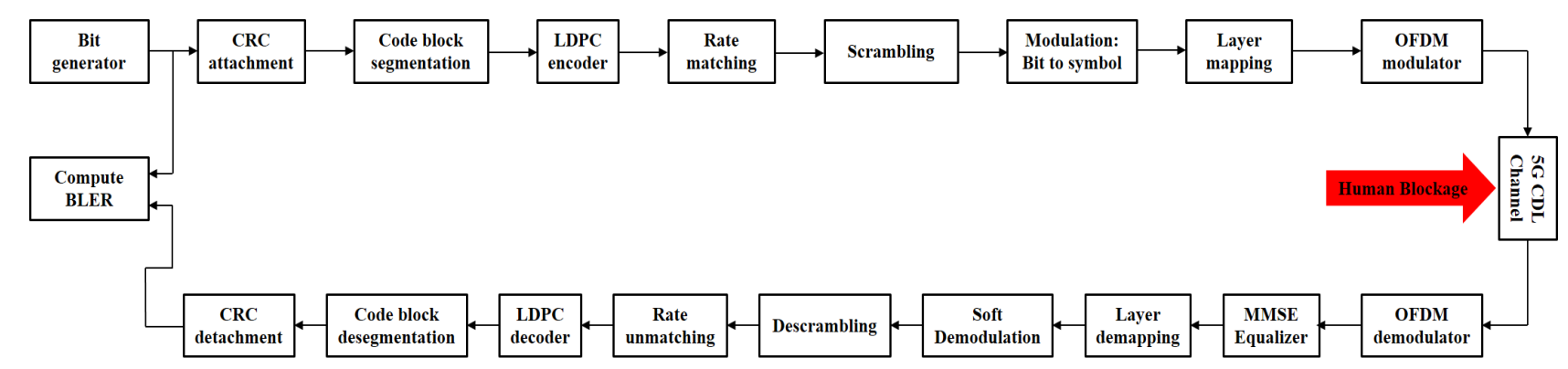

Fig. 4. Communication block diagram of $5 \mathrm{G}$ new radio.

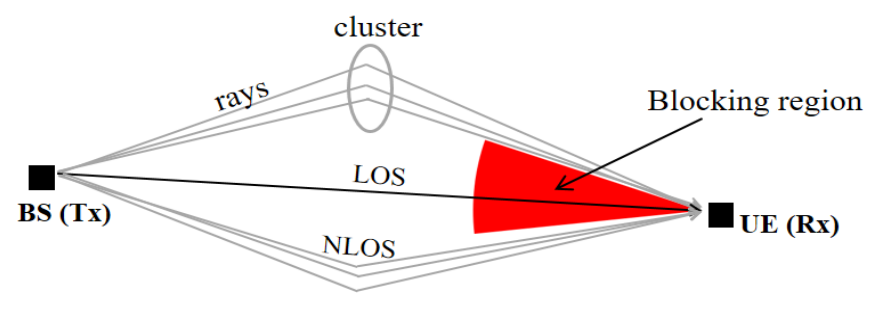

Fig. 5. 3GPP Blockage scenario.

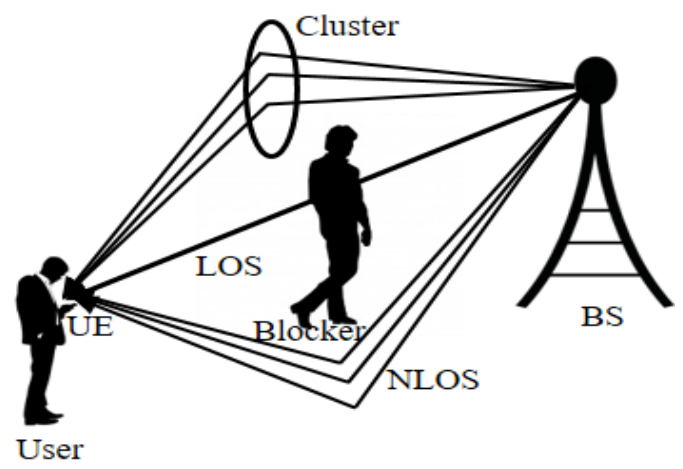

Fig. 6. Non-self-blocking illustration.

the blockage of the single beam direction. This mitigates the advantage of using mmWaves bands. In this scenario, MCS 2 is used, i.e QPSK modulation with a code rate of 0.3 .

However, in scenario 3 with the use of an additional second beam at the $\mathrm{Tx}$, an improvement in throughput close to scenario 1 is observed, going from $227.9 \mathrm{Mbits} / \mathrm{s}$ to 1.15 Gbits/s in the situation of blockage where MCS 2 is changed

TABLE II

SIMULATION PARAMETERS

\begin{tabular}{|c|c|}
\hline Parameters & Values \\
\hline Carrier Frequency & $60 \mathrm{GHz}$ \\
\hline Bandwidth & $400 \mathrm{MHz}$ \\
\hline Subcarrier Spacing & $120 \mathrm{kHz}$ \\
\hline Fast Fourier Transform (FFT) Size & 4096 \\
\hline Multipath Channel Model & 3GPP CDL-C \\
\hline Human Blockage Model & 3GPP Non-self-blocking \\
\hline Number of blockers & 1 \\
\hline
\end{tabular}

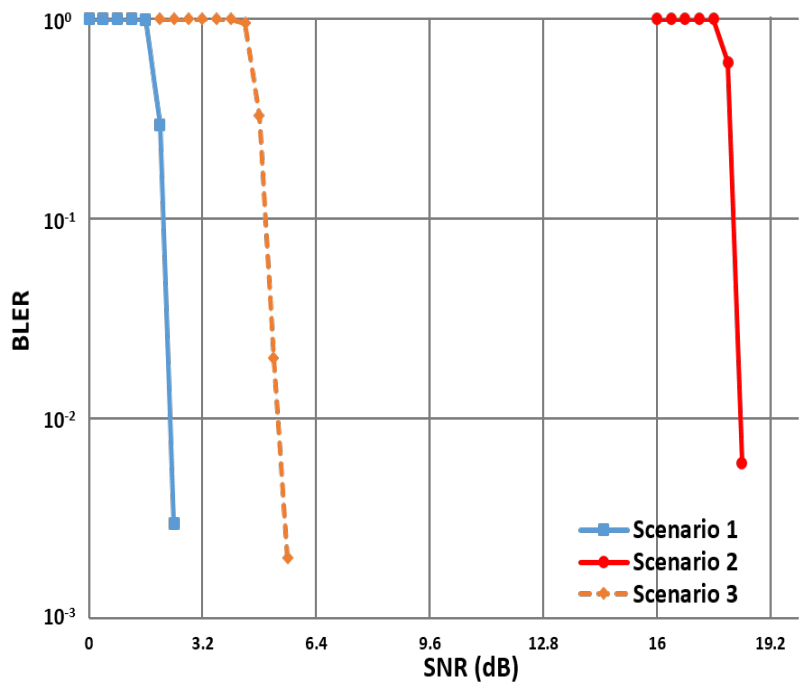

Fig. 7. Performance under the same throughput.

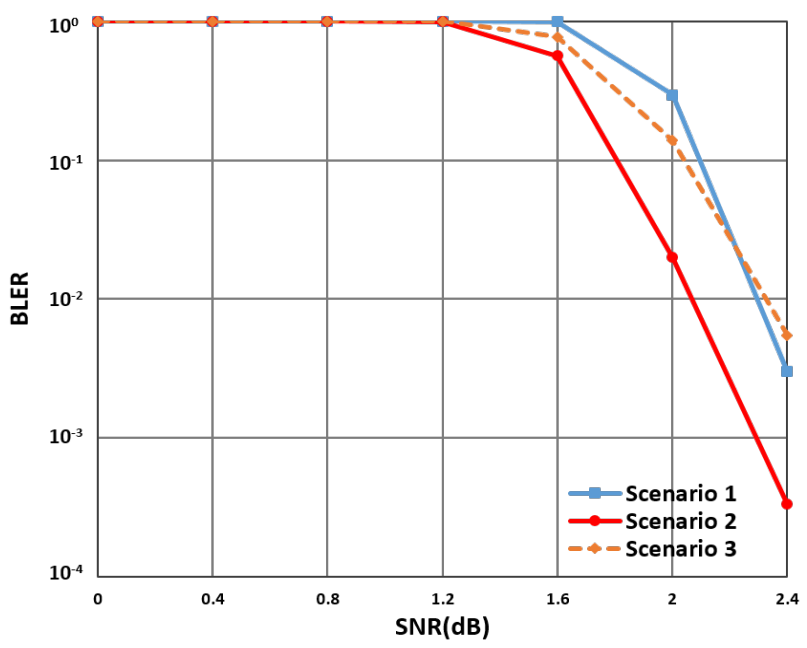

Fig. 8. Performance with three different throughputs.

for MCS 13 (64QAM with a code rate of 0.55). In other words, the blockage induces a loss of about $85 \%$ of the throughput, whereas with the MBA this loss is only $23 \%$. 


\section{CONCLUSION}

Multibeam antennas (MBAs) are able to generate independent and concurrent narrow beams pointing in different directions. They are low profile, cost effective and amenable for the human blockage resolution to keep the link-on-move in contrast to conventional beam steering mechanisms using multi-antennas systems which present implementation complexity and high hardware costs in mmWave bands. It is in this perspective that a generic approach is proposed in this paper for the MBA modelling. The proposed solution is flexible and can be used in the mmWave channel modelling for simulation purposes instead of using the $8 \mathrm{dBi}$ static 3GPP model defined for mmWave bands up to $100 \mathrm{GHz}$. Its application shows a quite good agreement with existing MBAs in terms of scan loss and beamwidth. However, the discrepancies become significant for the scan angle from $\pm 45^{\circ}$ off the antenna broadside due the increase in the spill-over radiation that is not considered in the proposed model.

For future work, we will extend the proposed MBA approach to the integration of the spill-over radiation effect from which the increase in the sidelobe level is considerable for high scan angles. In the same way, the impact of the unit-cells of TA or RA could also be investigated in the extension of the model. As we also deal with the 3GPP human blockage model, an improvement can be envisaged in order to make next studies and simulations more amenable.

\section{REFERENCES}

[1] S. Doğan, M. Karabacak and H. Arslan, "Optimization of Antenna Beamwidth under Blockage Impact in Millimeter-Wave Bands," in 2018 IEEE 29th Annual International Symposium on Personal, Indoor, and Mobile Radio Communications (PIMRC). Bologna, Italy, IEEE, December 2018.

[2] I. Uchendu and J. Kelly, "Survey of Beam Steering Techniques Available for Millimeter Wave Applications," Progress In Electromagnetics Research B, vol. 68, pp. 35-54, May 2016.

[3] A. B. Smolders, A. Reniers, U. Johannsen, and M. Herben, "Measurement and calibration challenges of microwave and millimeter-wave phased-arrays," in 2013 International Workshop on Antenna Technology (iWAT). Karlsruhe, Germany: IEEE, March 2013.

[4] R. T. Prabu, M. Benisha, V. Bai, and V. Yokesh, "Millimeter wave for 5G mobile communication application," in 2016 2nd International Conference on Advances in Electrical, Electronics, Information, Communication and BioInformatics (AEEICB). Chennai, India: IEEE, February 2016.

[5] G.-B. Wu, S.-W. Qu, and S. Yang, "Wide-Angle Beam-Scanning Reflectarray With Mechanical Steering," IEEE Transactions on Antennas and Propagation, vol. 66, no. 1, pp. 172-181, January 2018.

[6] W. Hong, Z. H. Jiang, C. Yu, J. Zhou, P. Chen, Z. Yu, H. Zhang, B. Yang, X. Pang, M. Jiang, Y. Cheng, M. K. T. Al-Nuaimi, Y. Zhang, J. Chen, and S. He, "Multibeam Antenna Technologies for 5G Wireless Communications," IEEE Transactions on Antennas and Propagation, vol. 65, no. 12, pp. 6231-6249, December 2017.

[7] 3GPP, "Technical Specification Group Radio Access Network; Study on channel model for frequencies from 0.5 to $100 \mathrm{GHz}$," Tech. Rep., June 2018, 3GPP TS 38.901 V15.0.

[8] IUT-R, "Guidelines for evaluation of radio interface technologies for IMT-Advanced," Tech. Rep., December 2009, Report ITU-R M.2135-1.

[9] E. C. DuFort, "Optimum networks for simultaneous multiple beam antennas," IEEE Transactions on Antennas and Propagation, vol. 40, no. 1 , pp. 1-7, January 1992.

[10] K. T. Pham, G. Liu, D. G. Ovejero, and R. Sauleau, "Dual-Band Transmitarray with Low Scan Loss for Satcom Applications," IEEE Transactions on Antennas and Propagation, October 2020.
[11] L. Eyraud, G. Grange, and H. Ohanessian, Théorie et Techniques des Antennes. Librairie Vuibert, PARIS, 1973.

[12] S. K. Karki, "Beam-Steerable E-Band Lens Antenna for 5G Backhaul Link," Master's thesis, Aalto University, October 2016.

[13] C.-T. Tai and C. Pereira, "An approximate formula for calculating the directivity of an antenna," IEEE Transactions on Antennas and Propagation, vol. 24, no. 2, pp. 235-236, March 1976.

[14] W. D. White, "Pattern limitations in multi-beam antennas," IRE Transactions on Antennas and Propagation, vol. AP-10, no. 4, pp. 430-436, July 1962.

[15] C. A. Balanis, Antenna Theory: Analysis and Design. Wiley, January 2005.

[16] L. Di Palma, A. Clemente, L. Dussopt, R. Sauleau, P. Potier and P. Pouliguen, "Circularly-Polarized Reconfigurable Transmitarray in KaBand With Beam Scanning and Polarization Switching Capabilities," IEEE Transactions on Antennas and Propagation, vol. 65, no. 2, pp. 529-540, February 2017.

[17] T. Pham, K. Pham, F. Gallée, and R. Sauleau, "A Wide-Angle BeamSteering Bifocal Transmitarray Antenna for 5G Channel Sounding at V-band," IEEE Transactions on Antennas and Propagation (submitted).

[18] F. Diaby, "Reconfigurable transmitarrays for beamsteering and beamforming at millimeterwaves," Ph.D. dissertation, Communauté Université Grenoble Alpes, December 2018.

[19] M. Jacob, C. Mbianke, and T. Kürner, "Human Body Blockage Guidelines for TGad MAC development," Tech. Rep., November 2009, IEEE 802.11- 09/1169r0.

[20] G. Z. S. Collonge and G. Zein, "Influence of the human activity on wide-band characteristics of the $60 \mathrm{GHz}$ indoor radio channel," IEEE Transactions on Wireless Communications, vol. 3, no. 6, pp. 2396-2406, November 2014.

[21] V. Raghavan, T. Bai, A. Sampath, O. H. Koymen and J. Li, "Modeling and Combating Blockage in Millimeter Wave Systems," in 2018 IEEE 19th International Workshop on Signal Processing Advances in Wireless Communications (SPAWC), Kalamata, Greece, June 2018.

[22] H. Dembélé, M. Le Bot, F. Gallée and P. Pajusco, "Impact of Human Blockage on 5G Communication System in the $26 \mathrm{GHz}$ Band," in 15th European Conference on Antennas and Propagation, Düsseldorf, Germany, March, 2021.

[23] 3GPP, "Technical Specification Group Radio Access Network; NR; Physical layer procedures for data," Tech. Rep., June 2019, 3GPP TS 38.214 V15.6.0.

[24] 3GPP, "Technical Specification Group Radio Access Network; NR; Base Station (BS) radio transmission and reception," Tech. Rep., September 2018, 3GPP TS 38.104 V15.3.0. 